\title{
Modulation of Host Biology by Pseudomonas aeruginosa Quorum Sensing Signal Molecules: Messengers or Traitors
}

\author{
Yi-Chia Liu', Kok-Gan Chan² and Chien-Yi Chang ${ }^{3,4 *}$ \\ ${ }^{1}$ Division of Molecular Microbiology, School of Life Sciences, University of Dundee, Dundee, UK, ${ }^{2}$ Division of Genetics and \\ Molecular Biology, Institute of Biological Sciences, Faculty of Science, University of Malaya, Kuala Lumpur, Malaysia, ${ }^{3}$ Centre \\ for Bacterial Cell Biology, Medical School, Newcastle University, Newcastle upon Tyne, UK, ${ }^{4}$ Interdisciplinary Computing and \\ Complex BioSystems (ICOS) Research Group, School of Computing Science, Newcastle University, Newcastle upon Tyne, \\ UK
}

OPEN ACCESS

Edited by:

Katharina F. Kubatzky, University Hospital Heidelberg,

Germany

Reviewed by:

Rodolfo García-Contreras, Instituto Nacional de Cardiologia,

Mexico

Gertrud Maria Hänsch,

University of Heidelberg, Germany

*Correspondence:

Chien-Yi Chang

chien-yi.chang@ncl.ac.uk

Specialty section

This article was submitted to Microbial Immunology, a section of the journal

Frontiers in Microbiology

Received: 09 September 2015

Accepted: 20 October 2015

Published: 09 November 2015

Citation:

Liu Y-C, Chan K-G and Chang C-Y (2015) Modulation of Host Biology by

Pseudomonas aeruginosa Quorum Sensing Signal Molecules: Messengers or Traitors.

Front. Microbiol. 6:1226.

doi: 10.3389/fmicb.2015.01226
Bacterial cells sense their population density and respond accordingly by producing various signal molecules to the surrounding environments thereby trigger a plethora of gene expression. This regulatory pathway is termed quorum sensing (QS). Plenty of bacterial virulence factors are controlled by QS or QS-mediated regulatory systems and QS signal molecules (QSSMs) play crucial roles in bacterial signaling transduction. Moreover, bacterial QSSMs were shown to interfere with host cell signaling and modulate host immune responses. QSSMs not only regulate the expression of bacterial virulence factors but themselves act in the modulation of host biology that can be potential therapeutic targets.

Keywords: Quorum sensing, $N$-acyl homoserine lactones, Pseudomonas quinolone signal, Pseudomonas aeruginosa, immunomodulation

\section{INTRODUCTION}

Quorum sensing (QS) is coined to describe the phenomenon of an intercellular co-operative behavior of bacteria used to coordinate the activities of individual cells. Diffusible QS signal molecules (QSSMs) play crucial roles in signal transduction of which, when QSSMs reach a threshold concentration, can coordinate multiple gene expression and a change in the behavior of bacterial population through the activation of sensor regulatory proteins (Fuqua et al., 1994; Miller and Bassler, 2001; Williams and Cámara, 2009). Bacteria produce a broad-range of signal molecules. Different types of QSSMs have been identified and characterized (LaSarre and Federle, 2013). Besides prokaryote, bacterial QSSMs also affect the settlement and germination of eukaryotic seaweed zoospores (Joint et al., 2002; Twigg et al., 2013). In several pathogenic bacteria, QS control their virulence determinants and contribute to bacterial pathogenesis. Due to the fact that the population density-dependent regulatory systems used by many bacterial pathogens are not essential for survival under most conditions, the disruption/interference of QS is considered an alternative approach to attenuate bacterial virulence in infections (LaSarre and Federle, 2013). However, this point has recently been argued that the resistance mechanisms against QS inhibitors could be possible and have been identified (Defoirdt et al., 2010, 2013; García-Contreras et al., 2013, 2015a). 
Pseudomonas aeruginosa is an ubiquitous Gram-negative bacterium with remarkably large and complex genome and is capable of adapting to versatile environments. In human cystic fibrosis (CF) lungs where $P$. aeruginosa has evolved the ability to form biofilms which are difficult to be eradicated by antibiotics (Heeb et al., 2011; Winsor et al., 2011). QS is responsible for the regulation of a large number of genes, for instance, around $10 \%$ of genes in the genome of $P$. aeruginosa are regulated by QS (Williams and Cámara, 2009). Here we review recent advances of $P$. aeruginosa QSSMs focusing on their roles in interference with host cells (Table 1) and the development of novel compounds that counteract the QSSMs activities.

\section{$N$-ACYL HOMOSERINE LACTONES (AHLS) AND THEIR MODULATIONS IN HOST CELLS}

Gram-negative bacteria, like Aliivibrio fischeri (previous Vibrio fischeri; Urbanczyk et al., 2007), have a conserved QS system with two central components, the LuxR-type and LuxI-type proteins, which serve as the signal receptor and signal synthase, respectively. LuxI catalyzes the synthesis of signaling molecules called $\mathrm{N}$-acyl homoserine lactones (AHLs). When an AHLs concentration of $10 \mathrm{nM}$ is reached, AHLs interact with LuxR and form a complex which promotes the expression of target genes, $\operatorname{lu} x \mathrm{ICDABE}$ for bioluminescence production and also the LuxI production (Kaplan and Greenberg, 1985). This forms a positive loop to produce more signal molecules (Fuqua et al., 1994; Cámara et al., 2002; Fuqua and Greenberg, 2002). The $N$-acyl homoserine lactone consists of a homoserine lactone ring from $S$-adenosylmethionine (SAM) and acyl chain from acyl acyl-carrier-protein (acyl-ACP) linked by an amide bond (Parsek et al., 1999). Based on the acyl-ACP binding site, different LuxI homologs produce different AHLs with various acyl side chains (Watson et al., 2002; Gould et al., 2004). A broad range of AHLs is produced in Gram-negative bacteria and AHL-QS systems control various bacterial behaviors (LaSarre and Federle, 2013). In $A$. fischeri $N$-(3-oxohexanoyl) homoserine lactone (3-oxo-C6-HSL) is produced for controlling bioluminescence production. In $P$. aeruginosa two AHL synthases, RhlI and LasI, produce a wide spectrum of AHLs including $N$-butanoylhomoserine lactone (C4-HSL), $N$-hexanoyl-homoserine lactone (C6-HSL) by RhlI and $N$-(3-oxooctanoyl)-homoserine lactone (3-oxo-C8-HSL), $\quad N$-(3-oxodecanoyl)-homoserine lactone (3-oxo-C10-HSL), $\mathrm{N}$-(3-oxododecanoyl)-homoserine lactone (3-oxo-C12-HSL) and N-(3-oxotetradecanoyl)-homoserine lactone (3-oxo-C14-HSL) by LasI (Ortori et al., 2011). An unusual $N$-(3-oxohexadecanoyl)-homoserine lactone (3oxo-C16-HSL) secreted by an environmental Pseudomonas sp. from a diseased Tilapia fish suggests that 3-oxo-C16HSL may contribute to the pathogenesis (Chang et al., 2012).

The abundant concentration of 3-oxo-C12-HSL in the culture of $P$. aeruginosa prompted investigations for its role in the pathogenesis with a mechanism potentially distinct from other pathogens. Indeed, 3-oxo-C12-HSL was found to activate mammalian cells through a mechanism independent of the tolllike receptor (TLR) pathways (Kravchenko et al., 2006). 3-oxoC12-HSL was shown to activate pro-inflammatory responses in human epithelial and fibroblast cells through the induction the transcriptional factor, nuclear factor kappa-light-chainenhancer of activated B cells (NF- $\kappa \mathrm{B})$ via the phosphorylation of ERK/MARK (Smith et al., 2001). However, this molecule selectively disrupts the NF- $\mathrm{B}$ signaling pathway in activated macrophages (Kravchenko et al., 2008). Studies indicated that 3oxo-C12-HSL not only induces apoptosis in haematopoietic cells but is cytototoxic to non-haematopoietic cells including airway epithelial cells, endothelial cells, fibroblasts, and mesenchymal stem cells (Tateda et al., 2003; Shiner et al., 2006; Schwarzer et al., 2010, 2012; Grabiner et al., 2014; Holban et al., 2014). $P$. aeruginosa 3-oxo-C12-HSL also impairs the epithelial barrier integrity through the alternations of calcium signaling and phosphorylation status of junctional proteins in the intestinal epithelial cells (Vikström et al., 2009, 2010).

In addition to its cytotoxicity, the role of 3-oxo-C12HSL in immunomodulation has been intensively investigated (Table 1). Ritchie et al. (2005) reported that 3-oxo-C12-HSL inhibits the differentiation of Th1 and Th2 cells. Human polymorphonuclear neutrophils (PMNs) are attracted by 3oxo-C12-HSL and increasingly express the adhesion proteins CD11b/CD18 and the immunoglobulin receptors CD16 and CD64 (Zimmermann et al., 2006; Wagner et al., 2007). The downregulation of the immune responses by 3-oxo-C12-HSL was demonstrated in human monocytes and murine macrophage-like cells in the presence of lipopolysaccharides (LPS) that 3-oxoC12-HSL inhibits the production of pro-inflammatory cytokine tumor necrosis factor $\alpha(\mathrm{TNF}-\alpha)$ but promotes the production of anti-inflammatory cytokine interleukin-10 (IL-10; Hooi et al., 2004; Glucksam-Galnoy et al., 2013). Grabiner et al. (2014) noticed that despite the increasing transcriptional expression of the murine interleukin 8 (IL-8) homologs $\mathrm{KC}$ and interleukin 6 (IL-6) in murine embryonic fibroblasts (MEFs), KC and IL-6 protein secretion were inhibited by the treatment of 3-oxo-C12HSL. It was shown that 3-oxo-C12-HSL acts upon the activation of endoplasmic reticulum (ER) stress transducer protein kinase RNA-like ER kinase (PERK) leading to the inhibition of protein synthesis. However, PERK is independent of 3-oxo-C12-HSL induced apoptosis indicating that 3-oxo-C12-HSL interferes with host cell biological activities through different mechanisms (Grabiner et al., 2014). Recent advances on the interactions between 3-oxo-C12-HSL and various types of host cells are highlighted in the review (Holm and Vikström, 2014).

Several host targets of 3-oxo-C12-HSL have been identified (Figure 1). In murine fibroblasts and human lung epithelial cells peroxisome proliferator-activated receptor beta/delta $(\operatorname{PPAR} \beta / \delta)$ and PPAR $\gamma$ may be the 3-oxo-C12-HSL receptors for proinflammatory responses (Jahoor et al., 2008; Cooley et al., 2010). 3-oxo-C12-HSL interacts and co-localizes with the IQ-motif-containing GTPase-activating protein IQGAP1 in human intestinal epithelial cells that causes the alternation of cell migration in a Racl and Cdc42- dependent manner (Karlsson et al., 2012). MEFs in lack of a transcriptional factor X-box binding protein 1 transcription factor (XBP1s) are 


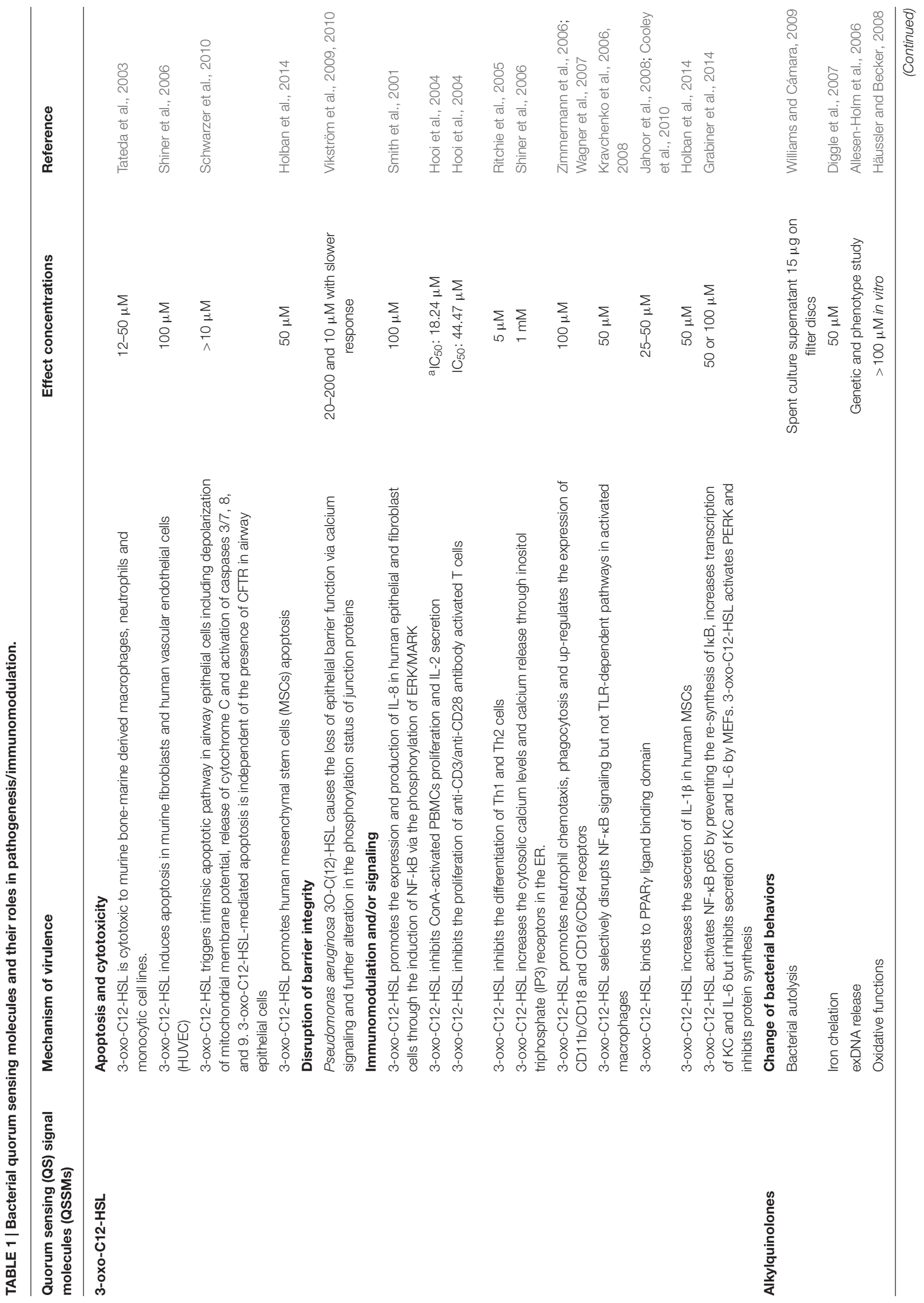


protective from 3-oxo-C12-HSL and C14-HSL ( $\mathrm{N}$-tetradecanoylhomoserine lactone) mediated apoptosis indicating that XBP1s is a critical host target in response of AHLs (Valentine et al., 2013). Paraoxonase 2, in response to 3 -oxo-C12-HSL through its lactonase activity, leads to apoptosis in human and murine embryotic epithelial cells (Schwarzer et al., 2015). Interestingly, 3oxo-C12-HSL activates the expression of a taste receptor T2R38 on the surface of primary human sinonasal cells (Lee et al., 2014) and neutrophils (Maurer et al., 2015). This recognition regulates calcium-dependent NO production thereby stimulates the mucociliary clearance and antibacterial effects suggesting an alternative innate immune defense mechanism distinct from the activation by canonical pattern recognition receptors (PRRs; Lee et al., 2012, 2014). Identification of the host compartments targeted by QSSMs could be the milestone for developing effective therapeutic methods against infections.

\section{PATHOGENIC ROLES OF ALKYL-QUINOLONE SIGNALS}

Pseudomonas aeruginosa also employs the alkyl-quinolone (AQ)-based QS system and the signal molecule was termed Pseudomonas quinolone signal (PQS; Pesci et al., 1999). The study of the AQs began from their intriguing structures similar to antimicrobial quinolones, although AQs were found no antimicrobial activities. Further studies unveiled that among more than 50 alkyl-quinolones found in $P$. aeruginosa, 2heptyl-3-hydroxy-4-(1H)-quinolone (PQS) and its precursor molecular 2-heptyl-4(1H)-quinolone (HHQ) are major QSSMs that cooperates with the AHL-QS (Xiao et al., 2006; Heeb et al., 2011). Synthesis of PQS depends on the pqsABCDE operon. PqsA, the anthranilate co-enzyme A ligase, catalyzes anthranilate that is produced by $\mathrm{PhnAB}$ to anthraniloyl-coenzyme A. PqsD mediates the synthesis of 2-aminobenzoylacetate (2-ABA) from anthraniloyl-coenzyme A and malonyl-CoA, decarboxylating coupling of 2-ABA to an octanoate group of octanoic acid that linked to PqsBC to produce HHQ (Dulcey et al., 2013). A recent study suggested PqsE is involved in the HHQ synthesis through hydrolysing the 2-ABA-CoA to form 2-ABA (Drees and Fetzner, 2015). HHQ can be transformed to PQS by the mono-oxygenase PqsH encoded by $p q s H$ located elsewhere on the chromosome (Pesci et al., 1999; Diggle et al., 2006). PqsR, also known as MvfR, is a LysR-type transcriptional regulator, with a conserved N-terminal DNA-binding helix-turn-helix and a C-terminal co-inducer-binding domain. PqsR activates the transcription of $p q s A B C D E$ and possibly the $p h n A B$ operon when binding to PQS or HHQ and triggers the typical QS autoinducing response enhancing $\mathrm{AQ}$ biosynthesis (Maddocks and Oyston, 2008; Heeb et al., 2011). PQS has been shown to reach the maximal production at late logarithmic phase (Diggle et al., 2003) and its production is promoted by the availability of the substrate anthranilate and the presence of aromatic amino acids (Palmer et al., 2005). AQ- and AHLQS in $P$. aeruginosa are hierarchical and involved in the regulation of multiple virulence factors including rhamnolipids, pyocyanin, elastases, exotoxin $\mathrm{A}$, and alkaline protease (Xiao 


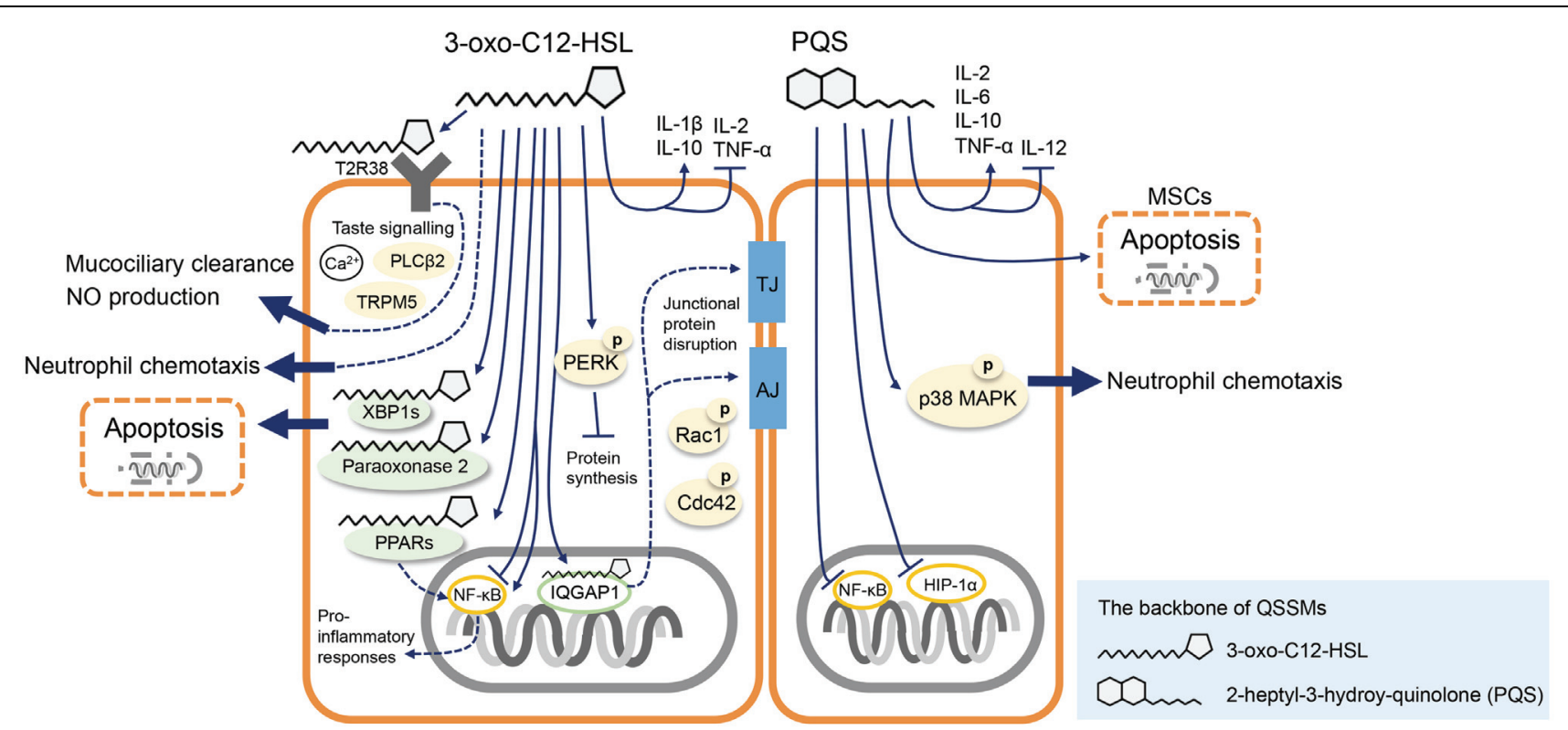

FIGURE 1 | Schematic illustration of the best characterized Pseudomonas aeruginosa quorum sensing signal molecules (QSSMs) that interfere with mammalian host biological functions. P. aeruginosa 3-oxo-C12-HSL targets XBP1 and paraoxonase 2 leading to host cell apoptosis, and the binding of 3-oxo-C12-HSL with IQGAP1 impairs the host cell integrity. 3-oxo-C12-HSL modulates innate immune responses via the activation of T2R38 receptor and inhibition of NF-kB pathways. P. aeruginosa Pseudomonas quinolone signal (PQS) molecule induces MSCs apoptosis, induces the secretion of inflammatory cytokines via the inhibition NF-kB and HIP-1 $\alpha$ pathways. PQS molecule interferes with neutrophils chemotaxis potentially through the activation of p38 MAPK pathways. See text for details. PLCß2, phospholipase C $\beta 2$; TRPM5, transient receptor potential cation channel subfamily M member 5; PPARs, peroxisome proliferator-activated receptors; TJ, tight junction; AJ, adhesional junction; MAPK, mitogen-activated protein kinase; MSCs, mesenchymal stem cells.

et al., 2006; Dubern and Diggle, 2008; Nadal Jimenez et al., 2012).

Pseudomonas quinolone signal is considered a multifunctional molecule. PQS is involved in bacterial cell autolysis at high population densities in nutrient deprived conditions (Williams and Cámara, 2009). PQS also has iron-chelating properties that contribute to iron transport and facilitates siderophoremediated iron delivery (Diggle et al., 2007). It has been demonstrated that there is far less extracellular DNA (exDNA) released by a $p q s A$ mutant than its wild-type counterpart either in planktonic or biofilm cultures (Allesen-Holm et al., 2006). PQS has dual pro- and anti-oxidative functions for developing different levels of tolerance in $P$. aeruginosa cells to environmental stress (Häussler and Becker, 2008). This may shape the whole population structure, increase the fitness in hostile environments and lead to the development of resistance to host immune systems (García-Contreras et al., 2015a,b).

A new QS molecule, 2-(2-hydroxyphenyl)-thiazole-4carbaldehyde (IQS) encoded by the $a m b B C D E$ operon was discovered recently (Lee et al., 2013). IQS is induced when $P$. aeruginosa is exposed to a phosphate-deprived environment. Under this unfavorable environment, expression of IQS overcomes the las-led QS circuit and promotes the expression of virulence factors. This finding may partially explain how $P$. aeruginosa clinical isolates persist in CF respiratory infections in the absence of a functional las system. Despite the fact that the AHL-QS of $P$. aeruginosa have been shown to play central roles in the regulation of virulence and immune modulation in vitro, this situation could differ in vivo. Around $50 \%$ of strains isolated from lungs of late stage CF patients are deficient in las $R$ function (Winstanley and Fothergill, 2009). Moreover, abolishing the whole AHL-QS by generating a quadruple mutation of rhlIR and lasIR exerted comparable infectivity to the wild-type strain in a mouse lung infection model (Lazenby et al., 2013), suggesting that the AHL-QS may not be required for full pathogenesis in vivo and other regulatory mechanism could be involved.

The contribution of the AQ-QS system to $P$. aeruginosa virulence was firstly described by Cao et al. (2001). PqsR positively regulates the expression of $p h n A B$ operon and the production of elastase, 3-oxo-C12-HSL and PQS that promotes the production of numerous virulence determinants. The $p q s R$ mutant was attenuated up to 320 -fold in the Arabidopsis plant infection model and caused a $65 \%$ reduction of mortality in a murine burn wound model (Cao et al., 2001). The interaction of PqsR with the AHL-QS was investigated by other groups showed that the effect of $p q s R$ deficiency on pathogenesis is independent from LasRI/RhlRI (Déziel et al., 2004; Dubern and Diggle, 2008). Mutations in the multidrug efflux pump, such as mexI and opmD led to the inhibition of PQS production and the attenuation of $P$. aeruginosa in rat and plant infection models. Provision of exogenous AQs to these mutants restored the virulence on plants (Aendekerk, 2005). Rampioni et al. (2010) found that both $p q s A$ and $p q s E$ mutants in PAO1 were attenuated in plant, nematode and mouse burn wound infection models. In an acute urinary tract infection model, PQS molecules were 
present in the renal and bladder tissue of mice infected with wild-type $P$. aeruginosa but absent in the mice with PQS mutants infections (Bala et al., 2014). Wild-type P. aeruginosa caused more severe inflammation and tissue destruction and greater levels of inflammatory cytokines TNF- $\alpha$, IL-6, and IL-10 at the site of infection in mice infected with wild-type strain than with PQS mutants. The virulence of PQS mutants can be restored by the addition of exogenous PQS molecules (Bala et al., 2014). These findings indicate that PQS participates in the pathogenesis of $P$. aeruginosa.

Pseudomonas quinolone signal has been identified in sputum, bronchoalveolar lavage fluid (BAL) and mucopurulent fluid from distal airways of end-stage CF lungs removed for transplant and at different stages from asymptotic early stage to late progression, suggesting a potential role of PQS in coordinating virulence factors during the course of infections (Collier et al., 2002; Guina et al., 2003). A study involving $60 \mathrm{CF}$ patients with chronic $P$. aeruginosa infection indicated that the AQs were detectable in the sputum, plasma and urine and the concentrations of molecules are positively correlated to the $P$. aeruginosa bacterial cell density. 2-nonyl-4-hydroxy-quinoline (NHQ) in plasma was suggested to be the biomarker for $P$. aeruginosa infection in CF lungs (Barr et al., 2015). An in vitro transcriptomic study investigating the physiology of $P$. aeruginosa grown in CF sputum revealed that the genes associated with PQS metabolism, such as those coding for the aromatic amino acid aminotransferase, 4-hydroxyphenylpyruvate dioxygenase $(h p d)$ and $p q s A B C D E$, were expressed 10-fold greater than the expression when $P$. aeruginosa was cultured in media containing glucose alone as the carbon source (Palmer et al., 2005).

Pseudomonas aeruginosa AQ molecules have been implicated in the immuno-modulation on host cells. PQS was shown to modulate cell proliferation, the production of interleukin2 (IL-2) and TNF- $\alpha$ in mitogen-stimulated human peripheral blood mononuclear cells (PBMCs; Hooi et al., 2004). PQS inhibited the production of IL-12 by LPS-stimulated bone marrow-derived dendritic cells which led to reduced T-cell proliferation (Skindersoe et al., 2009). Additionally AQ extracts derived from $P$. aeruginosa PA14 supernatants down-regulated host innate immune responses via inhibition of the NF- $\kappa B$ and hypoxia-inducible factor 1 alpha (HIF-1 $\alpha$ ) pathways in murine macrophages and cells obtained from BAL (Kim et al., 2010; Legendre et al., 2012). A recent study addressed the importance of timing in neutrophil infiltration in relation to the role of PQS in interference with neutrophil chemotaxis. Low levels of PQS stimulated the chemotaxis of neutrophils via the MAPK and p38 signaling pathways, whereas high levels of PQS, most likely produced by biofilm-like $P$. aeruginosa, did not interfere with neutrophils phagocytic capability and viability (Hänsch et al., 2014). Massive neutrophil accumulation is commonly seen in CF airways and high levels of neutrophil elastase correlate with poor pulmonary functions (Downey et al., 2008; Gifford and Chalmers, 2014). PQS may thus provide $P$. aeruginosa with another strategy for bacterial survival via the interference in multiple aspects of host biological activities.

\section{THE DEVELOPMENT OF INHIBITORS AND VACCINES AGAINST QSSMS}

Due to the fact that QSSMs have been implicated in the involvement of pathogenesis, the search for inhibitors and the development of vaccines that antagonize QSSMs are currently intensively investigated. Chang et al. (2014) suggested a strategy to screen novel anti-QS compounds from plant extraction that potentially could tackle the QS-mediated infections. The antibody 3-oxo-C12-HSL-BSA conjugate was also shown to alleviate the inflammatory responses by $P$. aeruginosa infections in an acute murine lung infection model (Miyairi et al., 2006). In a burn wound infection model, mice immunized with the vaccine 3-oxo-C12-HSL-r-PcrV conjugate before $P$. aeruginosa infection had higher survival than those without immunization (Golpasha et al., 2015). A high-throughput screening approach based on the inhibition of C12-mediated host responses identified triazolo[4,3-a]quinolines as 3-oxo-C12-HSL inhibitors with nanomolar potency that restore NF- $\kappa \mathrm{B}$ activity in 3-oxoC12-HSL treated cell lines and shown protective using an in vivo dermal infection model (Valentine et al., 2014). Since anthranilate (AA) being the precursor of AQs, halogenated AA analogs were found to inhibit the AQ biosynthesis and down-regulate the expression of PqsR controlled genes. Treatment with AA analogs prior to $P$. aeruginosa infection increased mice survival and lowered the bacterial dissemination to the organs (Lesic et al., 2007). MvfR-regulon inhibitors that bind QS transcriptional regulator MvfR (PqsR) were not only protective in murine acute and persistent infections against $P$. aeruginosa but also effectively reduced the formation of antibiotic-tolerant persisters (Starkey et al., 2014). These studies suggest the therapeutic potential of inhibitors and vaccines against QSSMs in both acute and chronic infections.

\section{CONCLUSION}

Quorum sensing-based bacterial communication links the individual bacterial cells to behave as multicellular organisms by employing signal molecules and to promote its population survival in the environment or hosts. QSSMs also interact with host cell signal pathways and the modulation of immune cell biology. For more than a decade strategies have been proposed from the use of inhibitors of QS for containing chronic infections (Hentzer et al., 2003) to the application of QSSMs for modulating immune responses to bacterial infections (Hancock et al., 2012). Understanding how QSSMs interact with host cells seems the promising land to tackle bacterial infections. Here we discussed recent advances on the interference of QSSMs with mammalian cells, the recently identified receptors on mammalian cells that target QSSMs and the QSSM inhibitors and their mechanisms. However, contradictory results suggested that many unknown mechanisms in complex bacteria-host interactions are remained. Also concern about bacterial resistance to QS inhibitors (GarcíaContreras et al., 2015a) and unexpected impact of QS inhibitors to environment (Decho et al., 2010) is increasing. Extending our understanding of the multiple roles of QSSMs would be valuable 
in the development of new therapeutic strategies against bacterial infections.

\section{AUTHOR CONTRIBUTIONS}

Y-CL, K-GC, C-YC wrote the paper. Y-CL made the figure. Y-CL and $\mathrm{C}-\mathrm{YC}$ made the table.

\section{REFERENCES}

Aendekerk, S. (2005). The MexGHI-OpmD multidrug efflux pump controls growth, antibiotic susceptibility and virulence in Pseudomonas aeruginosa via 4-quinolone-dependent cell-to-cell communication. Microbiology 151, 11131125. doi: 10.1099/mic.0.27631-0

Allesen-Holm, M., Barken, K. B., Yang, L., Klausen, M., Webb, J. S., Kjelleberg, S., et al. (2006). A characterization of DNA release in Pseudomonas aeruginosa cultures and biofilms. Mol. Microbiol. 59, 1114-1128. doi: 10.1111/j.13652958.2005.05008.x

Bala, A., Chhibber, S., and Harjai, K. (2014). Pseudomonas quinolone signalling system: a component of quorum sensing cascade is a crucial player in the acute urinary tract infection caused by Pseudomonas aeruginosa. Int. J. Med. Microbiol. 304, 1199-1208. doi: 10.1016/j.ijmm.2014.08.013

Barr, H. L., Halliday, N., Cámara, M., Barrett, D. A., Williams, P., Forrester, D. L., et al. (2015). Pseudomonas aeruginosa quorum sensing molecules correlate with clinical status in cystic fibrosis. Eur. Respir. J. 46, 1046-1054. doi: $10.1183 / 09031936.00225214$

Cámara, M., Williams, P., and Hardman, A. (2002). Controlling infection by tuning in and turning down the volume of bacterial small-talk. Lancet Infect. Dis. 2, 667-676. doi: 10.1016/S1473-3099(02)00447-4

Cao, H., Krishnan, G., Goumnerov, B., Tsongalis, J., Tompkins, R., and Rahme, L. G. (2001). A quorum sensing-associated virulence gene of Pseudomonas aeruginosa encodes a LysR-like transcription regulator with a unique selfregulatory mechanism. Proc. Natl. Acad. Sci. U.S.A. 98, 14613-14618. doi: 10.1073/pnas.251465298

Chang, C.-Y., Koh, C.-L., Sam, C.-K., Chan, X.-Y., Yin, W. F., and Chan, K. G. (2012). Unusual long-chain $\mathrm{N}$-acyl homoserine lactone production by and presence of quorum quenching activity in bacterial isolates from diseased tilapia fish. PLoS ONE 7:e44034. doi: 10.1371/journal.pone.0044034.t002

Chang, C.-Y., Krishnan, T., Wang, H., Chen, Y., Yin, W. F., Chong, Y. M., et al. (2014). Non-antibiotic quorum sensing inhibitors acting against $\mathrm{N}$-acyl homoserine lactone synthase as druggable target. Sci. Rep. 4:7245. doi: 10.1038/srep07245

Collier, D. N., Anderson, L., McKnight, S. L., Noah, T. L., Knowles, M., Boucher, R., et al. (2002). A bacterial cell to cell signal in the lungs of cystic fibrosis patients. FEMS Microbiol. Lett. 215, 41-46. doi: 10.1111/j.1574-6968.2002.tb11367.x

Cooley, M. A., Whittall, C., and Rolph, M. S. (2010). Pseudomonas signal molecule 3-oxo-C12-homoserine lactone interferes with binding of rosiglitazone to human PPAR $\gamma$. Microbes Infect. 7, 231-237. doi: 10.1016/j.micinf.2009. 12.009

Decho, A. W., Norman, R. S., and Visscher, P. T. (2010). Quorum sensing in natural environments: emerging views from microbial mats. Trends Microbiol. 18, 73-80. doi: 10.1016/j.tim.2009.12.008

Defoirdt, T., Boon, N., and Bossier, P. (2010). Can bacteria evolve resistance to quorum sensing disruption? PLoS Pathogens 6:e1000989. doi: 10.1371/journal.ppat.1000989.t001

Defoirdt, T., Brackman, G., and Coenye, T. (2013). Quorum sensing inhibitors: how strong is the evidence? Trends Microbiol. 21, 619-624. doi: 10.1016/j.tim.2013.09.006

Déziel, E., Gopalan, S., Tampakaki, A. P., Lépine, F., Padfield, K. E., Saucier, M., et al. (2004). The contribution of MvfR to Pseudomonas aeruginosa pathogenesis and quorum sensing circuitry regulation: multiple quorum sensing-regulated genes are modulated without affecting lasRI, rhlRI or the production of N-acyl- 1-homoserine lactones. Mol. Microbiol. 55, 998-1014. doi: $10.1111 / \mathrm{j} .1365-2958.2004 .04448 . x$

\section{ACKNOWLEDGMENTS}

We are grateful for the Open Access Funding from the University of Dundee. This work was also supported by the University of Malaya High Impact Research Grants (UM C/625/1/HIR/MOHE/CHAN/01, A-000001-50001, and UM C/625/1/HIR/MOHE/CHAN/14/1, H-50001-A000027) awarded to K-GC.

Diggle, S. P., Cornelis, P., Williams, P., and Cámara, M. (2006). 4-Quinolone signalling in Pseudomonas aeruginosa: old molecules, new perspectives. Int. J. Med. Microbiol. 296, 83-91. doi: 10.1016/j.ijmm.2006.01.038

Diggle, S. P., Matthijs, S., Wright, V. J., Fletcher, M. P., Chhabra, S. R., Lamont, I. L., et al. (2007). The Pseudomonas aeruginosa 4-quinolone signal molecules HHQ and PQS play multifunctional roles in quorum sensing and iron entrapment. Chem. Biol. 14, 87-96. doi: 10.1016/j.chembiol.2006.11.014

Diggle, S. P., Winzer, K., Chhabra, S. R., Worrall, K. E., Cámara, M., and Williams, P. (2003). The Pseudomonas aeruginosa quinolone signal molecule overcomes the cell density-dependency of the quorum sensing hierarchy, regulates rhl-dependent genes at the onset of stationary phase and can be produced in the absence of LasR. Mol. Microbiol. 50, 29-43. doi: 10.1046/j.13652958.2003.03672.x

Downey, D. G., Bell, S. C., and Elborn, J. S. (2008). Neutrophils in cystic fibrosis. Thorax 64, 81-88. doi: 10.1136/thx.2007.082388

Drees, S. L., and Fetzner, S. (2015). PqsE of Pseudomonas aeruginosa acts as pathway-specific thioesterase in the biosynthesis of alkylquinolone signaling molecules. Chem. Biol. 22, 611-618. doi: 10.1016/j.chembiol.2015.04.012

Dubern, J.-F., and Diggle, S. P. (2008). Quorum sensing by 2-alkyl-4-quinolones in Pseudomonas aeruginosa and other bacterial species. Mol. BioSyst. 4, 882-888. doi: 10.1039/b803796p

Dulcey, C. E., Dekimpe, V., Fauvelle, D.-A., Milot, S., Groleau, M.-C., Doucet, N., et al. (2013). The end of an old hypothesis: the Pseudomonas signaling molecules 4-hydroxy-2-alkylquinolines derive from Fatty Acids, not 3-ketofatty acids. Chem. Biol. 20, 1481-1491. doi: 10.1016/j.chembiol.2013.09.021

Fuqua, C., and Greenberg, E. P. (2002). Signalling: listening in on bacteria: acyl-homoserine lactone signalling. Nat. Rev. Mol. Cell Biol. 3, 685-695. doi: 10.1038/nrm907

Fuqua, W. C., Winans, S. C., and Greenberg, E. P. (1994). Quorum sensing in bacteria: the LuxR-LuxI family of cell density-responsive transcriptional regulators. J. Bacteriol. 176, 269-275.

García-Contreras, R., Maeda, T., and Wood, T. K. (2013). Resistance to quorum-quenching compounds. Appl. Environ. Microbiol. 79, 6840-6846. doi: 10.1128/AEM.02378-13

García-Contreras, R., Maeda, T., and Wood, T. K. (2015a). Can resistance against quorum-sensing interference be selected? ISME J. doi: 10.1038/ismej.2015.84

García-Contreras, R., Nuñez-López, L., Jasso-Chávez, R., Kwan, B. W., Belmont, J. A., Rangel-Vega, A., et al. (2015b). Quorum sensing enhancement of the stress response promotes resistance to quorum quenching and prevents social cheating. ISME J. 9, 115-125. doi: 10.1038/ismej.2014.98

Gifford, A. M., and Chalmers, J. D. (2014). The role of neutrophils in cystic fibrosis. Curr. Opin. Hematol. 21, 16-22. doi: 10.1097/MOH.0000000000000009

Glucksam-Galnoy, Y., Sananes, R., Silberstein, N., Krief, P., Kravchenko, V. V., Meijler, M. M., et al. (2013). The bacterial quorum-sensing signal molecule N3-oxo-dodecanoyl-L-homoserine lactone reciprocally modulates pro- and antiinflammatory cytokines in activated macrophages. J. Immunol. 191, 337-344. doi: 10.4049/jimmunol.1300368

Golpasha, I. D., Mousavi, S. F., Owlia, P., Siadat, S. D., and Irani, S. (2015). Immunization with 3-oxododecanoyl-L-homoserine lactone-r-PcrV conjugate enhances survival of mice against lethal burn infections caused by Pseudomonas aeruginosa. Bosn. J. Basic Med. Sci. 15, 15-24. doi: 10.17305/bjbms.2015.292

Gould, T. A., Schweizer, H. P., and Churchill, M. E. A. (2004). Structure of the Pseudomonas aeruginosa acyl-homoserinelactone synthase LasI. Mol. Microbiol. 53, 1135-1146. doi: 10.1111/j.1365-2958.2004.04211.x

Grabiner, M. A., Fu, Z., Wu, T., Barry, K. C., Schwarzer, C., and Machen, T. E. (2014). Pseudomonas aeruginosa quorum-sensing molecule homoserine lactone 
modulates inflammatory signaling through PERK and eI-F2. J. Immunol. 193, 1459-1467. doi: 10.4049/jimmunol.1303437

Guina, T., Purvine, S. O., Yi, E. C., Eng, J., Goodlett, D. R., Aebersold, R., et al. (2003). Quantitative proteomic analysis indicates increased synthesis of a quinolone by Pseudomonas aeruginosa isolates from cystic fibrosis airways. Proc. Natl. Acad. Sci. U.S.A. 100, 2771-2776. doi: 10.1073/pnas.0435846100

Hancock, R. E. W., Nijnik, A., and Philpott, D. J. (2012). Modulating immunity as a therapy for bacterial infections. Nat. Rev. Microbiol. 10, 243-254. doi: 10.1038/nrmicro2745

Hänsch, G. M., Prior, B., Brenner-Weiss, G., Obst, U., and Overhage, J. (2014). The Pseudomonas quinolone signal (PQS) stimulates chemotaxis of polymorphonuclear neutrophils. JABFM 12, 21-26. doi: 10.5301/jabfm.5000204

Häussler, S., and Becker, T. (2008). The Pseudomonas quinolone signal (PQS) balances life and death in Pseudomonas aeruginosa populations. PLoS Pathogens 4:e1000166. doi: 10.1371/journal.ppat.1000166.s002

Heeb, S., Fletcher, M. P., Chhabra, S. R., Diggle, S. P., Williams, P., and Cámara, M. (2011). Quinolones: from antibiotics to autoinducers. FEMS Microbiol. Rev. 35, 247-274. doi: 10.1111/j.1574-6976.2010.00247.x

Hentzer, M., Wu, H., Andersen, J. B., Riedel, K., Rasmussen, T. B., Bagge, N., et al. (2003). Attenuation of Pseudomonas aeruginosa virulence by quorum sensing inhibitors. EMBO J. 22, 3803-3815. doi: 10.1093/emboj/cdg366

Holban, A.-M., Bleotu, C., Chifiriuc, M. C., Bezirtzoglou, E., and Lazar, V. (2014). Role of Pseudomonas aeruginosa quorum sensing (QS) molecules on the viability and cytokine profile of human mesenchymal stem cells. Virulence 5, 303-310. doi: 10.4161/viru.27571

Holm, A., and Vikström, E. (2014). Quorum sensing communication between bacteria and human cells: signals, targets, and functions. Front. Plant Sci. 5:309. doi: 10.3389/fpls.2014.00309

Hooi, D. S. W., Bycroft, B. W., Chhabra, S. R., Williams, P., and Pritchard, D. I. (2004). Differential immune modulatory activity of Pseudomonas aeruginosa quorum-sensing signal molecules. Infect. Immun. 72, 6463-6470. doi: 10.1128/IAI.72.11.6463-6470.2004

Jahoor, A., Patel, R., Bryan, A., Do, C., Krier, J., Watters, C., et al. (2008). Peroxisome proliferator-activated receptors mediate host cell proinflammatory responses to Pseudomonas aeruginosa autoinducer. J. Bacteriol. 190, 4408-4415. doi: 10.1128/JB.01444-07

Joint, I., Tait, K., Callow, M. E., Callow, J. A., Milton, D., Williams, P., et al. (2002). Cell-to-cell communication across the prokaryote-eukaryote boundary. Science 298, 1207. doi: 10.1126/science. 1077075

Kaplan, H. B., and Greenberg, E. P. (1985). Diffusion of autoinducer is involved in regulation of the Vibrio fischeri luminescence system. J. Bacteriol. 163, 1210-1214.

Karlsson, T., Turkina, M. V., Yakymenko, O., Magnusson, K.-E., and Vikström, E. (2012). The Pseudomonas aeruginosa N-acylhomoserine lactone quorum sensing molecules target IQGAP1 and modulate epithelial cell migration. PLoS Pathogens 8:e1002953. doi: 10.1371/journal.ppat.1002953

Kim, K., Kim, Y. U., Koh, B. H., Hwang, S. S., Kim, S.-H., Lépine, F., et al. (2010). HHQ and PQS, two Pseudomonas aeruginosa quorum-sensing molecules, down-regulate the innate immune responses through the nuclear factorkappaB pathway. Immunology 129, 578-588. doi: 10.1111/j.1365-2567.2009.0 3160.x

Kravchenko, V. V., Kaufmann, G. F., Mathison, J. C., Scott, D. A., Katz, A. Z., Grauer, D. C., et al. (2008). Modulation of gene expression via disruption of NF-KB signaling by a bacterial small molecule. Science 321, 259-263. doi: $10.1126 /$ science. 1156499

Kravchenko, V. V., Kaufmann, G. F., Mathison, J. C., Scott, D. A., Katz, A. Z., Wood, M. R., et al. (2006). N-(3-Oxo-acyl) homoserine lactones signal cell activation through a mechanism distinct from the canonical pathogenassociated molecular pattern recognition receptor pathways. J. Biol. Chem. 281, 28822-28830. doi: 10.1074/jbc.M606613200

LaSarre, B., and Federle, M. J. (2013). Exploiting quorum sensing to confuse bacterial pathogens. Microbiol. Mol. Biol. Rev. 77, 73-111. doi: 10.1128/MMBR.00046-12

Lazenby, J. J., Griffin, P. E., Kyd, J., Whitchurch, C. B., and Cooley, M. A. (2013). A quadruple knockout of lasIR and rhliR of Pseudomonas aeruginosa PAO1 that retains wild-type twitching motility has equivalent infectivity and persistence to PAO1 in a mouse model of lung infection. PLoS ONE 8:e60973. doi: 10.1371/journal.pone.0060973
Lee, J., Wu, J., Deng, Y., Wang, J., Wang, C., Wang, J., et al. (2013). A cellcell communication signal integrates quorum sensing and stress response. Nat. Chem. Biol. 9, 339-343. doi: 10.1038/nchembio.1225

Lee, R. J., Chen, B., Redding, K. M., Margolskee, R. F., and Cohen, N. A. (2014). Mouse nasal epithelial innate immune responses to Pseudomonas aeruginosa quorum-sensing molecules require taste signaling components. Innate Immun. 20, 606-617. doi: 10.1177/1753425913503386

Lee, R. J., Xiong, G., Kofonow, J. M., Chen, B., Lysenko, A., Jiang, P., et al. (2012). T2R38 taste receptor polymorphisms underlie susceptibility to upper respiratory infection. J. Clin. Invest. 122, 4145-4159. doi: 10.1172/JCI 64240

Legendre, C., Reen, F. J., Mooij, M. J., McGlacken, G. P., Adams, C., and O'Gara, F. (2012). Pseudomonas aeruginosa alkyl quinolones repress hypoxia-inducible factor 1 (HIF-1) signaling through HIF-1 $\alpha$ degradation. Infect. Immun. 80, 3985-3992. doi: 10.1128/IAI.00554-12

Lesic, B., Lépine, F., Déziel, E., Zhang, J., Zhang, Q., Padfield, K., et al. (2007). Inhibitors of pathogen intercellular signals as selective anti-infective compounds. PLoS Pathogens 3:e126. doi: 10.1371/journal.ppat.0030126.st003

Maddocks, S. E., and Oyston, P. C. F. (2008). Structure and function of the LysR-type transcriptional regulator (LTTR) family proteins. Microbiology 154, 3609-3623. doi: 10.1099/mic.0.2008/022772-0

Maurer, S., Wabnitz, G. H., Kahle, N. A., Stegmaier, S., Prior, B., Giese, T., et al. (2015). Tasting Pseudomonas aeruginosa biofilms: human neutrophils express the bitter receptor T2R38 as sensor for the quorum sensing molecule N-(3-oxododecanoyl)-1-Homoserine Lactone. Front. Immunol. 6:369. doi: 10.3389/fimmu.2015.00369

Miller, M. B., and Bassler, B. L. (2001). Quorum sensing in bacteria. Ann. Rev. Microbiol. 55, 165-199. doi: 10.1146/annurev.micro.55.1.165

Miyairi, S., Tateda, K., Fuse, E. T., Ueda, C., Saito, H., Takabatake, T., et al. (2006). Immunization with 3-oxododecanoyl-L-homoserine lactone-protein conjugate protects mice from lethal Pseudomonas aeruginosa lung infection. J. Med. Microbiol. 55, 1381-1387. doi: 10.1099/jmm.0.46658-0

Nadal Jimenez, P., Koch, G., Thompson, J. A., Xavier, K. B., Cool, R. H., and Quax, W. J. (2012). The multiple signaling systems regulating virulence in Pseudomonas aeruginosa. Microbiol. Mol. Biol. Rev. 76, 46-65. doi: 10.1128/MMBR.05007-11

Ortori, C. A., Dubern, J.-F., Chhabra, S. R., Cámara, M., Hardie, K., Williams, P., et al. (2011). Simultaneous quantitative profiling of N-acyl-L-homoserine lactone and 2-alkyl-4(1H)-quinolone families of quorum-sensing signaling molecules using LC-MS/MS. Anal. Bioanal. Chem. 399, 839-850. doi: 10.1007/s00216-010-4341-0

Palmer, K. L., Mashburn, L. M., Singh, P. K., and Whiteley, M. (2005). Cystic fibrosis sputum supports growth and cues key aspects of Pseudomonas aeruginosa physiology. J. Bacteriol. 187, 5267-5277. doi: 10.1128/JB.187.15.5267-5277.2005

Parsek, M. R., Val, D. L., Hanzelka, B. L., Cronan, J. E., and Greenberg, E. P. (1999). Acyl homoserine-lactone quorum-sensing signal generation. Proc. Natl. Acad. Sci. U.S.A. 96, 4360-4365. doi: 10.1073/pnas.96.8.4360

Pesci, E. C., Milbank, J. B., Pearson, J. P., McKnight, S., Kende, A. S., Greenberg, E. P., et al. (1999). Quinolone signaling in the cell-to-cell communication system of Pseudomonas aeruginosa. Proc. Natl. Acad. Sci. U.S.A. 96, 1122911234. doi: 10.1073/pnas.96.20.11229

Rampioni, G., Pustelny, C., Fletcher, M. P., Wright, V. J., Bruce, M., Rumbaugh, K. P., et al. (2010). Transcriptomic analysis reveals a global alkyl-quinoloneindependent regulatory role for PqsE in facilitating the environmental adaptation of Pseudomonas aeruginosa to plant and animal hosts. Environ. Microbiol. 12, 1659-1673. doi: 10.1111/j.1462-2920.2010.02214.x

Ritchie, A. J., Jansson, A., Stallberg, J., Nilsson, P., Lysaght, P., and Cooley, M. A. (2005). The Pseudomonas aeruginosa quorum-sensing molecule N3-(oxododecanoyl)-L-homoserine lactone inhibits T-cell differentiation and cytokine production by a mechanism involving an early step in T-cell activation. Infect. Immun. 73, 1648-1655. doi: 10.1128/IAI.73.3.1648-165 5.2005

Schwarzer, C., Fu, Z., Morita, T., Whitt, A. G., Neely, A. M., Li, C., et al. (2015). Paraoxonase 2 serves a proapopotic function in mouse and human cells in response to the Pseudomonas aeruginosa quorum-sensing molecule N-(3-oxododecanoyl)-homoserine lactone. J. Biol. Chem. 290, 7247-7258. doi: 10.1074/jbc.M114.620039 
Schwarzer, C., Fu, Z., Patanwala, M., Hum, L., Lopez-Guzman, M., Illek, B., et al. (2012). Pseudomonas aeruginosa biofilm-associated homoserine lactone C12 rapidly activates apoptosis in airway epithelia. Cell. Microbiol. 14, 698-709. doi: 10.1111/j.1462-5822.2012.01753.x

Schwarzer, C., Wong, S., Shi, J., Matthes, E., Illek, B., Ianowski, J. P., et al. (2010). Pseudomonas aeruginosa homoserine lactone activates storeoperated cAMP and cystic fibrosis transmembrane regulator-dependent $\mathrm{Cl}$ secretion by human airway epithelia. J. Biol. Chem. 285, 34850-34863. doi: 10.1074/jbc.M110.167668

Shiner, E. K., Terentyev, D., Bryan, A., Sennoune, S., Martinez-Zaguilan, R., Li, G., et al. (2006). Pseudomonas aeruginosa autoinducer modulates host cell responses through calcium signalling. Cell. Microbiol. 8, 1601-1610. doi: 10.1111/j.1462-5822.2006.00734.x

Skindersoe, M. E., Zeuthen, L. H., Brix, S., Fink, L. N., Lazenby, J., Whittall, C., et al. (2009). Pseudomonas aeruginosa quorum-sensing signal molecules interfere with dendritic cell-induced T-cell proliferation. FEMS Immun. Med. Microbiol. 55, 335-345. doi: 10.1111/j.1574-695X.2008.00533.x

Smith, R. S., Fedyk, E. R., Springer, T. A., Mukaida, N., Iglewski, B. H., and Phipps, R. P. (2001). IL-8 production in human lung fibroblasts and epithelial cells activated by the Pseudomonas autoinducer N-3-oxododecanoyl homoserine lactone is transcriptionally regulated by NF-kappa B and activator protein-2. J. Immunol. 167, 366-374. doi: 10.4049/jimmunol.167.1.366

Starkey, M., Lépine, F., Maura, D., Bandyopadhaya, A., Lesic, B., He, J., et al. (2014). Identification of anti-virulence compounds that disrupt quorum-sensing regulated acute and persistent pathogenicity. PLoS Pathogens 10:e1004321. doi: 10.1371/journal.ppat.1004321.s009

Tateda, K., Ishii, Y., Horikawa, M., Matsumoto, T., Miyairi, S., Pechere, J. C., et al. (2003). The Pseudomonas aeruginosa autoinducer N-3-oxododecanoyl homoserine lactone accelerates apoptosis in macrophages and neutrophils. Infect. Immun. 71, 5785-5793. doi: 10.1128/IAI.71.10.5785-5793.2003

Twigg, M. S., Tait, K., Williams, P., Atkinson, S., and Cámara, M. (2013). Interference with the germination and growth of Ulva zoospores by quorumsensing molecules from Ulva-associated epiphytic bacteria. Environ. Microbiol. 16, 445-453. doi: 10.1111/1462-2920.12203

Urbanczyk, H., Ast, J. C., Higgins, M. J., Carson, J., and Dunlap, P. V. (2007). Reclassification of Vibrio fischeri, Vibrio logei, Vibrio salmonicida and Vibrio wodanis as Alivibrio fischeri gen. nov., comb. nov., Aliivibrio logei comb. nov., Aliivibrio salmonicida comb. nov. and Aliivibrio wodanis comb. nov. Int. J. Syst. Evol. Microbiol. 57, 2823-2829. doi: 10.1099/ijs.0.65081-0

Valentine, C. D., Anderson, M. O., Papa, F. R., and Haggie, P. M. (2013) $\mathrm{X}$-box binding protein 1 (XBP1s) is a critical determinant of Pseudomonas aeruginosa homoserine lactone-mediated apoptosis. PLoS Pathog. 9:e1003576. doi: 10.1371/journal.ppat.1003576

Valentine, C. D., Zhang, H., Phuan, P.-W., Nguyen, J., Verkman, A. S., and Haggie, P. M. (2014). Small molecule screen yields inhibitors of Pseudomonas homoserine lactone-induced host responses. Cell. Microbiol. 16, 1-14. doi: $10.1111 / \mathrm{cmi} .12176$
Vikström, E., Bui, L., Konradsson, P., and Magnusson, K.-E. (2009). The junctional integrity of epithelial cells is modulated by Pseudomonas aeruginosa quorum sensing molecule through phosphorylation-dependent mechanisms. Exp. Cell Res. 315, 313-326. doi: 10.1016/j.yexcr.2008.10.044

Vikström, E., Bui, L., Konradsson, P., and Magnusson, K.-E. (2010). Role of calcium signalling and phosphorylations in disruption of the epithelial junctions by Pseudomonas aeruginosa quorum sensing molecule. Eur. J. Cell Biol. 89, 584597. doi: 10.1016/j.ejcb.2010.03.002

Wagner, C., Zimmermann, S., Brenner-Weiss, G., Hug, F., Prior, B., Obst, U., et al. (2007). The quorum-sensing molecule N-3-oxododecanoyl homoserine lactone (3OC12-HSL) enhances the host defence by activating human polymorphonuclear neutrophils (PMN). Anal. Bioanal. Chem. 387, 481-487. doi: 10.1007/s00216-006-0698-5

Watson, W. T., Minogue, T. D., Val, D. L., von Bodman, S. B., and Churchill, M. E. A. (2002). Structural basis and specificity of acyl-homoserine lactone signal production in bacterial quorum sensing. Mol. Cell 9, 685-694. doi: 10.1016/S1097-2765(02)00480-X

Williams, P., and Cámara, M. (2009). Quorum sensing and environmental adaptation in Pseudomonas aeruginosa: a tale of regulatory networks and multifunctional signal molecules. Curr. Opin. Microbiol. 12, 182-191. doi: 10.1016/j.mib.2009.01.005

Winsor, G. L., Lam, D. K. W., Fleming, L., Lo, R., Whiteside, M. D., Yu, N. Y., et al. (2011). Pseudomonas genome database: improved comparative analysis and population genomics capability for Pseudomonas genomes. Nucleic Acids Res. 39, D596-D600. doi: 10.1093/nar/gkq869

Winstanley, C., and Fothergill, J. L. (2009). The role of quorum sensing in chronic cystic fibrosis Pseudomonas aeruginosa infections. FEMS Microbiol. Lett. 290, 1-9. doi: 10.1111/j.1574-6968.2008.01394.x

Xiao, G., He, J., and Rahme, L. G. (2006). Mutation analysis of the Pseudomonas aeruginosa mvfR and pqsABCDE gene promoters demonstrates complex quorum-sensing circuitry. Microbiology 152, 1679-1686. doi: 10.1099/mic.0.28605-0

Zimmermann, S., Wagner, C., Muller, W., Brenner-Weiss, G., Hug, F., Prior, B., et al. (2006). Induction of neutrophil chemotaxis by the quorum-sensing molecule N-(3-oxododecanoyl)-L-homoserine lactone. Infect. Immun. 74, 5687-5692. doi: 10.1128/IAI.01940-05

Conflict of Interest Statement: The authors declare that the research was conducted in the absence of any commercial or financial relationships that could be construed as a potential conflict of interest.

Copyright (C) 2015 Liu, Chan and Chang. This is an open-access article distributed under the terms of the Creative Commons Attribution License (CC BY). The use, distribution or reproduction in other forums is permitted, provided the original author(s) or licensor are credited and that the original publication in this journal is cited, in accordance with accepted academic practice. No use, distribution or reproduction is permitted which does not comply with these terms. 